\title{
Influence of deposition angle on the structural, morphological and optical properties of sputtered AlN thin films
}

Mahbobeh Ravankhah and Hadi Savaloni*

Department of Physics, University of Tehran, North-Kargar Street, Tehran, Iran

* Correspondence: savaloni@ khayam.ut.ac.ir; Tel.: +98 21 88635776; fax: +98 2188004781.

\begin{abstract}
Fabrication of AlN thin films of $90 \mathrm{~nm}$ thickness was performed using magnetron sputtering technique at different deposition angles of $0^{\circ}, 15^{\circ}, 30^{\circ}, 45^{\circ}$ and $60^{\circ}$. Structural and morphological characteristics of the produced samples were obtained by means of x-ray diffraction (XRD), atomic force microscopy (AFM) and field emission electron microscopy (FESEM) analyses. The spectrophotometry analysis with both s- and p-polarized lights at two incident light angles of $30^{\circ}$ and $60^{\circ}$ were carried out for obtaining the optical spectra of the samples while Kramers-Kronig method was used for calculation of refractive index and further analysis carried out to obtain energy gaps and absorption peaks. Results showed maximum void fraction and surface roughness for film deposited at $30^{\circ}$ consistent with previous publications on oblique angle deposition. The higher the porosity of the AlN films in this work showed lower refractive index and because smaller grains/nanoparticles are produced larger energy band gaps are obtained.
\end{abstract}

Keywords: AlN; Oblique angle deposition; Optical properties; Kramers-Kronig; XRD; AFM

\section{Introduction}

One dimensional structures such as nano-columns are anisotropic structures which can be produced using physical vapor deposition technique at oblique angle deposition (OAD). These structures because of their particular geometry are of interest to the researchers. This geometry can be controlled and affects all physical properties of the thin film. On the other hand, 
aluminum nitride because of its optical and electrical properties has many applications in electronics and optoelectronics. The intriguing features of aluminum nitride are its large band gap $(6.2 \mathrm{eV})$, large refractive index (2.0), high electrical resistivity (109-1011 $\Omega \mathrm{m})$ and high thermal conductivity $(180 \mathrm{mWm}-1 \mathrm{~K}-1)$. Different methods are used for fabrication of aluminum nitride such as chemical vapor deposition (CVD) [1], molecular beam epitaxy (MBE) [2], pulsed laser deposition (PLD) [3] and sputtering [4] while their optical and electrical properties are also investigated. Considering these properties and applications, fabrication of aluminum nitride in different nano-structural shapes such as nano-wires, nano-rods and nano-tubes is of high importance. Aluminum nitride nano-wires of 30-100 nm diameter and length of 500-700 nm by un-equilibrium plasma deposition technique at temperatures above $6000{ }^{\circ} \mathrm{C}$ is reported [5]. Also, hexagonal AlN nano-wires are fabricated at $1100{ }^{\circ} \mathrm{C}$ via reaction of $\mathrm{Al}$ powder with $\mathrm{NH}_{3}-\mathrm{N}_{2}$ [6] and AlN nano-rods using electron beam deposition and annealing process of $\mathrm{Al}_{2} \mathrm{~N}_{3} / \mathrm{BaS}$ thin film at $800-900{ }^{\circ} \mathrm{C}$ [7].

In this work we have produced AlN thin films with nano-rod structure using DC sputtering technique together with OAD method at five different deposition angles. The optical properties and structural characteristics of fabricated thin films are investigated. From the optical spectra measurements by s- and p-polarized incident lights at two incident angles of $30^{\circ}$ and $60^{\circ}$ and use of Kramers-Kronig theory optical properties and the energy band gaps of the produced samples are obtained and correlation with structural characteristics is achieved.

\section{Experimental details}

Aluminum nitride thin films of $90 \mathrm{~nm}$ thickness were coated on ultrasonically cleaned glass substrates (cut from microscope slide with dimensions $20 \mathrm{~mm} \times 20 \mathrm{~mm}$ ) by a DC reactive 
magnetron sputtering system using high purity circular $\mathrm{Al}$ targets $(99.98 \%)$ of $76 \mathrm{~mm}$ diameter and $1 \mathrm{~mm}$ thickness. The target to substrate distance was $10 \mathrm{~cm}$. The substrates were held at room temperature and at five different deposition angles of $0^{\circ}, 15^{\circ}, 30^{\circ}, 45^{\circ}$ and $60^{\circ}$. A continuously variable DC power supply of $800 \mathrm{~V}$ and $150 \mathrm{~mA}$ was used as power source for sputtering. The thicknesses and the deposition rate of these aluminum nitride films were checked in situ using a quartz crystal monitor (6 MHz gold, Inficon Company, USA) positioned near the substrate and at the same angle during the sputtering process. The sputtering chamber was pumped down to $2 \times 10^{-5}$ mbar using a turbo molecular pump prior to introduction of an $\mathrm{Ar} / \mathrm{N}_{2}$ gas mixture ( $4 \mathrm{sccm} \mathrm{Ar}+4 \mathrm{sccm} \mathrm{N}_{2}$ ). The flow rates of both argon and nitrogen gases were controlled individually by mass flow controllers. Two samples were prepared for each deposition angle in each run. One of the samples was used for the structural characterization and the other one was used for optical investigations. The deposition rate of the films at normal incidence angle was $10 \AA^{-1}$.

After deposition, structural and optical properties of the films were characterized. Nanostructure and crystallographic orientation of the samples were obtained using a Philips XRD X'pert MPD Diffractometer $\left(\mathrm{Cu} \mathrm{K} \mathrm{K}_{\alpha}\right.$ radiation) with a step size of $0.02^{\circ}$ and count time of $1 \mathrm{~s}$ per step. Surface morphology of the samples was studied using a field emission scanning electron microscope (FESEM) (CamScan MV2300, Czech and England). An atomic force microscope (AFM) (Auto Probe PC, Park Scien-tific Instrument, USA) was also used for investigation of the surface morphology and roughness of the samples. Measurements of the optical spectra of films was carried out by means of a Stellarnet EPP-2000 spectrophotometer in the wavelength region of $400-850 \mathrm{~nm}$ with both $\mathrm{s}$ and $\mathrm{p}$ linearly polarized light at two incident light angles of $30^{\circ}$ and $60^{\circ}$.

\section{Theory}


Kramers-Kronig (KK) analysis of the first type is related to the normal-incidence reflectivity spectra [8]. Although this approach breaks down for instance for off-normal reflectivity, or for a thin film on a substrate and in many other cases, but a number of analytical and numerical algorithms are suggested to extend the KK method to off-normal reflection [9-15], transmission [16-19], attenuated total reflection spectra [20-22] as well as to optical data on layered samples [23-27] and low-symmetry crystals [28,29] and non-standard situations. Hence, KK method is explained in many publications and here we briefly describe the method we have used in this work which can be applied to thin films. If we know the frequency dependence of the real part of an analytical function, then by using the KK relation between the real and the imaginary parts we can determine the frequency dependence of imaginary part and vice versa. The KK relations for the real part of refractive index is given in [30]:

where, $\omega$ is the angular frequency and P represents the Cauchy principal value of the integral.

From the reflection and transmission spectra of the sample and the use of Eq. 2 at each incident light wavelength the imaginary part of the refractive index, $k$ was calculated:

$$
\exp (-4 \pi k d / \lambda)=\frac{T}{1-R}
$$

In this paper, The Cauchy equations is used to model the refractive index in the spectral range where the films are transparent by using a Cauchy dispersion [31]:

$$
\begin{aligned}
& \mathrm{n}(\lambda)=\mathrm{A}_{\mathrm{n}}+\frac{\mathrm{B}_{\mathrm{n}}}{\lambda^{2}}+\frac{\mathrm{C}_{\mathrm{n}}}{\lambda^{4}}+\cdots \\
& \mathrm{k}(\lambda)=\mathrm{A}_{\mathrm{k}}+\frac{\mathrm{B}_{\mathrm{k}}}{\lambda^{2}}+\frac{\mathrm{C}_{\mathrm{k}}}{\lambda^{4}}+\cdots
\end{aligned}
$$

where $\mathrm{A}, \mathrm{B}$ and $\mathrm{C}$ are specific material parameters. 
Considering that the imaginary part of the refractive index is known only in a finite frequency region, between $\omega_{1}$ and $\omega_{2}$ one may assume;

$$
P \int_{0}^{\infty} \frac{\dot{\omega}}{\dot{\omega}^{2}-\omega^{2}} k(\dot{\omega}) d \dot{\omega} \approx P \int_{\omega_{1}}^{\omega_{2}} \frac{\dot{\omega}}{\dot{\omega}^{2}-\omega^{2}} k(\dot{\omega}) d \dot{\omega}
$$

Having the values of the imaginary part of the refractive index, one can easily calculate the real part for each wavelength by employing the KK method.

\section{Results and discussions}

\subsection{FESEM analyses}

In Fig. 1(a) to Fig. 1(e) the cross-section images of the AIN thin films deposited at five different deposition angles of $0^{\circ}, 15^{\circ}, 30^{\circ}, 45^{\circ}$ and $60^{\circ}$ are given, respectively. It is worthwhile to mention here that although the film thicknesses of $90 \mathrm{~nm}$ was deposited but the SEM cross-section images in Fig. 1(a) to Fig. 1(e) show much larger thicknesses. This can be due to the fact that the quartz crystal during the deposition process measured the mass deposited which was calibrated for fully dense AlN while the produced films are of reduced density.

The columnar structure of the films can be distinguished in these images and it can be seen that by increasing the deposition angle $(\alpha)$ the rise angle $(\beta)$ of the columns increases. The relationship between these two angles for the deposition angles less than 60 is given by the wellknown tangent law;

$\tan (\beta)=\frac{1}{2} \tan (\alpha)$

In Table 1 the results of tangent law and the values obtained experimentally from the cross section images of the samples (Fig. 1) are compared and relatively good agreement is obtained. 
In Fig. 1 it can be seen that the thickness of the film is decreased by increasing the deposition angle. This can be due to the fact that at normal deposition $\left(\alpha=0^{\circ}\right)$ the incident atom on the substrate surface on collision losses a large amount of energy and by a small amount of remaining energy may diffuse on the surface for a short distance, hence becoming accommodated on the substrate surface. On the other hand, for those atoms incident on the substrate surface at oblique angle this decomposes into two components: a parallel to, and a perpendicular to the substrate surface. Therefore, it can diffuse a longer distance on the substrate surface then it either joins to the pre-deposited atoms and produce a denser structure or reevaporate from the substrate surface. Both of these processes can reduce the film thickness. In addition at oblique angle deposition a lower amount of vapor flow reaches the substrate surface which can reduce the film thickness too.

\subsection{AFM results}

Fig. 2 shows the 3D and 2D AFM images (columns I and II) as well as surface void/inclusion fractions (column III), while in Table 2 the results of grain size distribution, percentage of surface void fraction (measured using JMicroVision software), average and root mean square surface roughness for all samples produced in this work obtained from the AFM results are given.

Results in Table 2 for the produced samples at different deposition angles show that the fraction of voids, surface roughness and the mean grain size are varied with deposition angle and there is a maximum value for all of these three parameters for the deposition angle of $30^{\circ}$. The structure zone model (SZM) [32-35] predicts that by increasing the deposition angle the void fraction should increase. Our results in previous work [36] and those of Takakura et al. [37,38] show a deviation from this general rule, that at a certain deposition angle (e.g. $30^{\circ}$ in this work and $45^{\circ}$ 
in [38]), the surface void fraction increases and then at higher deposition angles, it decreases. Takakura et al. [38] related this phenomenon to the rearrangement of adatoms resulting from the diffusion or thermal vibration. Siabi-Garjan et al. [36] related this phenomenon to the available sites on the substrate/growing film surface for relaxation of an adatom which depends on the underlying surface crystallography of the substrate or the growing film surface and surface energy, affects the catchment/diffusion length (diffusion effect) and this in turn should directly depend on the deposition angle of adatoms (flux). Therefore, the increase of void fraction at $30^{\circ}$ incidence angle in this work is in agreement with the above mentioned references and may be explained by the same statements. The increase of surface roughness at this deposition angle is also directly related to the increase of void fraction.

\subsection{X-ray diffraction analyses}

XRD patterns of all samples are given in Fig. 3. Apart from the film deposited at $0^{\circ}$ all other samples show amorphous-like pattern. This can be due to smallness of the coherently diffracting domains in the structure of the film which affects the scattering power of the incident x-ray. Even in case of the film produced at $0^{\circ}$ deposition angle suggestion of a small peak for $\operatorname{AlN}(100)$ is very speculative. The large hump at diffraction angles less than $40^{\circ}$ is characteristic of the glass substrate used in this work.

\subsection{Optical analyses}

The measured optical spectra obtained in the wavelength range of 400 to $850 \mathrm{~nm}$ for both s- and p- polarized incident lights on the samples at two angles of $30^{\circ}$ and $60^{\circ}$ are given in Fig. 4. It can be seen that the spectra obtained for s- and p-polarized lights at $30^{\circ}$ incident light angle show almost the same behaviour with variation of deposition angle. However when the incident light angle is increased to $60^{\circ}$ different behaviour is observed. 
As it is discussed in [39] when the incident light interacts with the columnar structure at an angle the $E_{P}$ field decomposes into two components: a field parallel $E_{P I I}$ to, and a field perpendicular $E_{p \perp}$ to the long axis of this structure. Therefore, the larger $\theta$ results in larger $E_{P I I}$ component. Therefore, the results of low incidence angle $\left(30^{\circ}\right)$ should contain information about the surface morphology of the film, including anisotropy when the $\varphi$ angle is changed, while high incidence angle $\left(60^{\circ}\right)$ interacts with the Mn column with its maximum electric field.

At near normal incidence both s- and p-electric fields are in the surface of the sample and orthogonal to each other and should produce the same reflection and transmission spectra. By increasing the incident light angle the s-field remains in the surface of the sample while as mentioned above the p-field decomposes with a component in the film surface and one perpendicular to the film surface, hence its optical spectra deviates from that of s-polarized incident light as it can be distinguished in Fig. 4. In addition, Khoshman [40] investigated the optical properties of sputtered aluminium nitride films using spectroscopic elipsometry and reported that the reflection intensity of s-polarized light increases with incident light angle. This effect can be distinguished in our work for two deposition angles of $0^{\circ}$ and $30^{\circ}$ when results of incident light angle of $60^{\circ}$ are compared with those of $30^{\circ}$ in Fig. 4.

The refractive index of the samples for both s- and p- incident polarized lights was obtained using the Kramers-Kronig method. From the reflection and transmission spectra of the sample and the use of Eq. 6 at each incident light wavelength the imaginary part of the refractive index, $k$ was calculated.

Having the values of the imaginary part of the refractive index, one can easily calculate the real part for each wavelength by employing the Kramers-Kronig method. Results for both s- and p- 
polarized lights and at two incident light angles of $30^{\circ}$ and $60^{\circ}$ are given in Fig. 5. In Fig. 5, it can be seen that the refractive index varies between 1.0 and 1.6 in the wavelength region examined in this work. The variation of these results with both incident light angle and polarization of light is due to the variation of anisotropy (bundling effect [41-44]) in the structure of the produced samples.

It is well known that the refractive index is lower for the porous structures relative to non-porous structures. Our samples in this work with nano-rod structure are porous, therefore it is expected that the obtained refractive indices be less than that for a single crystal [45]. It should also be considered that the film thickness also affects the obtained results. As it can be observed in Fig. 5 the refractive index of our samples are less than that for a single crystal and they decrease with increasing the wavelength of the incident light. This is consistent with the theoretical predictions and other experimental results. Joo et al. [46] produced AlN thin films of 948-1198 nm thickness on quartz substrates by reactive radio frequency magnetron sputtering method and obtained the refractive index of their samples in the wavelength region of 190-820 nm. The value of refractive index for their samples is reported between 1.95 and 2.5. They have also pointed out that the value of refractive index depends on the structure of the film as well as surface oxidation of the sample which decreases the refractive index. The other factor which causes reduction of the refractive index is the density of the film. The increased temperature during the film growth process increases the diffusion and mobility effects, hence denser films are produced. Watanabe et al. [47] investigated the influence of temperature on the refractive index of AIN thin films. They found that refractive index increases almost linearly with temperature except for the near band gap region (below $230 \mathrm{~nm}$ ). 
The energy gap of the samples produced in this work was obtained from the optical results using the extrapolation of the $(\alpha h v)^{2}$ plot versus $h v$ and fitting of higher energy part of the plot to its tangent. Results are given in Fig. 6. Different results are obtained for s- and p-polarized incident lights which can be due to the difference between transmission spectra as discussed in the preceding sections. The energy gap of a material depends on its porosity, thickness, particle/grain size and structure of thin film. Higher porosity leads to lower number of particles, hence reduced number of energy states and increased gap between valance and conductance bands which in turn increases the energy gap. The energy gap also increases with decrease of thickness and particle size. In this work by changing the deposition angle we have produced films with different porosity, thickness and surface roughness that all of them affect the energy gap. Hajiakbari et al. [48] reported an energy gap of $4.15 \mathrm{eV}$ and $4.10 \mathrm{eV}$ for amorphous AlN films of $539 \mathrm{~nm}$ and 675 $\mathrm{nm}$ thickness, respectively. Khoshman and Kordesch [40] reported band gap energy of $5.81 \mathrm{eV}$ for amorphous AlN thin film of $100 \mathrm{~nm}$ thickness.

In Fig. 7 variation of band gap energy versus deposition angle is given. It is expected that by increasing the deposition angle, hence reduction of film thickness and the size of the grains, the band gap energy increases. However, it can be seen that an oscillatory behavior is obtained with a minimum for the $30^{\circ}$ deposition angle and for both $30^{\circ}$ and $60^{\circ}$ incident light angles. In general, the energy gap depends on the chemical bounding of the elements composing the material and its crystallography as well as its surface morphology. Amorphous materials have a smaller band gap relative to crystallite materials [40]. Considering that amorphous-like structure is obtained for our samples (see XRD results in section 4.3) and knowing that increased density of nitrogen in the AlN structure can cause reduction of the energy gap [49,50] it may be expected that films produced at $30^{\circ}$ and $60^{\circ}$ deposition angles due to higher void fraction in their structure 
(Table 2) leading to higher effective surface area for reaction between $\mathrm{Al}$ atoms and the nitrogen atoms/ions, contain higher amount of nitrogen in their structure. Therefore, all these features may have been responsible for the decreased energy gap in these samples.

In Fig. 8 the absorption spectra of AlN thin films produced at three different incident light angles of $0^{\circ}, 30^{\circ}$, and $60^{\circ}$ measured in the wavelength region of 400 to $850 \mathrm{~nm}$ are given. In these spectra two main absorption peaks can be distinguished. The first peak is usually observed in AlN samples containing oxygen atoms [51] while the second peak that is located at the blue wavelength is obtained in most of AlN samples produced using different methods. It can be seen that by changing the deposition angle no change is occurred in the position of this peak (Fig. 8).

Oxygen is a common impurity in $\mathrm{AlN}$ and $\mathrm{Al}$ vacancies (VAl) and $\mathrm{N}$ vacancies (VN) are the most relevant defects. These defects and their complexes with oxygen may cause absorption band observed in AlN samples. The optical properties of defects in AlN have been studied using hybrid functional calculation, a computational method based on the density functional theory (DFT) by Yan et al. [51]. They found binding nitrogen vacancy in a positive charge state composed of $\mathrm{Al}$ vacancy and $\mathrm{O}$ impurity (VAl-ON), give rise to an absorption peak in $3.03 \mathrm{eV}$ $(410 \mathrm{~nm})$. The other absorption which occurs at $2.49 \mathrm{eV}(499 \mathrm{~nm})$ is due to transition between VAl and VN, $V_{A l}^{3-}+V_{N}^{+} \rightarrow V_{A l}^{2-}+V_{N}^{0}$. These two transitions agree very well with the values observed in this work.

\section{Conclusions}

The special optical properties observed for thin films produced using oblique angle deposition is caused by the anisotropy (bundling effect) in the structure of this type of thin films. By changing the deposition angle, the film surface roughness, porosity, film thickness and the size of the grains in the structure of the film changes. All of these parameters have direct effect on the 
optical spectra of the produced samples. AlN thin films were produced by magnetron sputtering technique at five different deposition angles of $0^{\circ}, 15^{\circ}, 30^{\circ}, 45^{\circ}$ and $60^{\circ}$. Their structures and morphology were analyzed using XRD, AFM and SEM while their optical properties were obtained from the measurements of the films optical spectra in the wavelength region of 400-850 $\mathrm{nm}$ with both s- and p-polarized incident lights at two incident light angles of $30^{\circ}$ and $60^{\circ}$. It was found that the porosity and film surface roughness increase with deposition angle up to a certain angle $\left(30^{\circ}\right)$ and at this angle they reach their maximum values which is consistent with the results reported in the literature and explained on the basis of rearrangement of atoms resulting from the diffusion or thermal vibration and the available sites on the substrate/growing film surface for relaxation of an adatom. This depends on the underlying surface crystallography of the substrate or the growing film surface and surface energy, and affects the catchment/diffusion length (diffusion effect), and this, in turn, directly should depend on the deposition angle of adatoms (flux). In studying the reflection and transmission spectra one should consider the influence of the incident light angle on the sample and its polarization state. By increasing the incident light angle the direction of s- and p-polarization electric fields changes relative to the sample which leads to obtaining different optical spectra which in turn results in different refractive indices for two different polarizations. For the porous films produced in this work as expected lower refractive indices are obtained relative to the single crystal sample. Also because of this porosity larger energy gaps are obtained. This is related to the formation of lower number of particles and their small sizes. In addition, the energy gap is also varied with deposition angle owing to the changes occurred in the morphology of the film. Hence, it can be concluded that the most important factor in studying the optical property of thin films and in particular in OAD is the geometry of the film which can be controlled by pre-design of the deposition method. 


\section{Acknowledgements}

This work was carried out with the support of the University of Tehran. H.S. is grateful to the Iran National Science Foundation (INSF) and the Centre of Excellence for Physics of Structure and Microscopic Properties of Matter, Department of Physics, University of Tehran, for partial support of this work. 


\section{References:}

1. S. C. Shi, C. F. Chen, S. C. Cgattopadhyay, K. H. Chen, Appl. Phys. Lett. 87 (2005) 073109.

2. K. A. Bertness, A. Roshko, N. A. Sanford, J. M. Barker, A. Davydov, J. Cryst. Growth 287 (2006) 522.

3. S. Six, B. Rauschenbach, Thin Solid Films, 415 (2002) 285.

4. H. Cheng, Y. Sun, J. X. Zhang, Y. B. Zhang, S. Yuan, P. Hing, J. Cryst. Growth, 254 (2003) 46.

5. C. Balasubramanian, V. P. Godbole, V. K. Rohatgi, A. K. Das, S. V. Bhoraskar, Nanotechnology, 15 (2004) 370.

6. Q. Wu, Z. Hu, X. Wang, Y. Lu, K. Huo, S. Deng, N. Xu, B. Shen, R. Zhang, Y. Chen, J. Mater. Chem., 13 (2003) 2024.

7. P. F. Smet, Jo E. Van Haecke, D. Poelman, Sol. State Commun., 139 (2006) 522.

8. F. C. Jahoda, Phys. Rev., 107 (1957) 1261.

9. H. S. Somal, PhD thesis, University of Groningen (1998).

10. G. M. Hale, W. E. Holland, and M. R. Querry, Appl. Opt., 12 (1973) 48.

11. E. G. Makarova and V. N. Morozov, Opt. Spectrosc., 40 (1976) 138.

12. L. I. Alperovich and V. N. Pushkarev, Opt. Spectrosc., 47 (1979) 516.

13. J. A. Bardwell and M. J. Dignam, J. Chem. Phys., 83 (1985) 5468.

14. P. Grosse and V. Offermann, Appl. Phys. A, Solids Surf. A52 (1991) 138.

15. V. Hopfe, P. Bussemer, E. Richter, and P. Klobes, J. Phys. D, Appl. Phys.25, 288 (1992).

16. J. D. Neufeld and G. Andermann, J. Opt. Soc. Am., 62 (1972) 1156. 
17. R. D. Bringans, J. Phys. D, Appl. Phys., 10 (1977) 1855.

18. K. Yamamoto and H. Ishida, Vib. Spectrosc., 15 (1997) 27.

19. C.-P. E. Varsamis, Appl. Spectrosc., 56 (2002) 1107.

20. A. E. Tshmel and V. I. Vettegren, Spectrochim. Acta A, Mol. Spectrosc., 29A (1973) 1681.

21. K. Yamamoto, A. Masui, and H. Ishida, Appl. Opt., 33 (1994) 6285.

22. J. E. Bertie and Lan Zhida, J. Chem. Phys.,105 (1996) 8502.

23. E. A. Lupashko, V. K. Miloslavskii, and I. N. Shklyarevskii, Opt. Spectrosc., 29 (1970) 419.

24. W. J. Plieth and K. Naegele, Surf. Sci., 50 (1975) 53.

25. A. V. Tikhonravov and I. V. Zuev, Mosc. Univ. Phys. Bull., 48 (1993) 44.

26. A. F. Santander-Syro, R. P. S. M. Lobo, N. Bontemps, Z. Konstantinovic, Z. Li, and H. Raffy, Phys. Rev. Lett., 88 (2002) 097005.

27. A. F. Santander-Syro, PhD thesis, ESPCI, Paris (2001).

28. E. E. Koch, A. Otto, and K. L. Kliewer, Chem. Phys., 3 (1974) 362.

29. A. B. Kuzmenko, E. A. Tishchenko, and A. S. Krechetov, Opt. Spectrosc., 84 (1998) 402.

30. V. Lucarini, J. J. Saarinen, V. E. Peiponen, E. M. Vartiainen, Kramers-Kronig Relations in Optical Materials Research, Springer, Berlin (2005).

31. D. Poelman, P. F. Smet, J. Appl. Phys., 36 (2003) 1850.

32. H. Savaloni and M. Gholipour-Shahraki, Phys. Stat. Sol. B 244 (2007) 3115.

33. L. Abelmane and C. Lodder, Thin Solid Films 305 (1997) 1.

34. H. Savaloni and M. Gholipour-Shahraki, Nanotechnology 15 (2004) 311. 
35. H. Savaloni, M. H. Reissi, M. Shariati and M. A. Player, Thin Solid Films 515 (2006) 439.

36. A. Siabi-Garjan, H. Savaloni, J. Beik-Mohammadi and A.-R. Grayeli-Korpi; Phil. Mag., 93 (2013) 3527.

37. Y. Ueda, W. Takakura and A. Yamada, J. Magn. Magn. Mater., 239 (2002) 45.

38. W. Takakura, S. Ikeda and Y. Ueda, Mater. Trans. 42 (2001) p.881.

39. H. Savaloni, F. Haydari-Nasab, M. Malmir, Appl. Surf. Sci., 257 (2011) 9044.

40. J. M. Khoshman, M. E. Kordesch, J. Non-Crystal. Sol., 351 (2005) 3334.

41. J.P. Singh, T.Karabacak,D.-X.Ye, andD.-L. Liup, J. Vac. Sci. Technol. B 23, 2114-2121 (2005). doi:10.1116/1.2052747. 698

42. L. Abelmann and C. Lodder, Thin Solid Films 305, 1 (1997).

43. J. Takadoum (Ed), Nanomaterials and Surface Engineering, ISTE Ltd 2010.

44. A. Siabi-Garjan, H. Savaloni, J. Beik-Mohammadi, and A.R. Grayeli-Korpi, Philos. Magn. 93, 3527 (2013).

45. J. Pastrnak and L. Roskovcova, Physica Status Solidi (b) 14(10) (1966) k5-k8. DOI: $10.1002 /$ pssb.19660140127

46. H. Y. Joo, H. J. Kim, S. J. Kim, S. Y. Kim, J. Vac. Sci. Technol. A 17 (1999) 862.

47. N. Watanabe, T. Kimoto, J. Suda, J. Appl. Phys. 104 (2008) 106101.

48. F. Hajiakbari, M. Mojtabazade Larijani, M. Ghorannevis, M.Aslaninejad, A. Hojabri, Jpn. J. Appl. Phys. 49 (2010) 095802.

49. R. K. Choudhary, P. Mishra, A. Biswas, A. C. Bidaye, ISRN Material Science 2013 (2013)

50. J. M. Khoshman, M. E. Kordesch, Phys. Stat. Sol. (C) 2 (2005) 2821. 
51. Q. Yan, A. Janotti, M. Scheffler, C. G. Van de Walle, Appl. Phys. Lett. 105 (2014) 111104.

\section{Figure captions:}

Figure 1. FESEM images of AIN thin films with nano-rod structure produced at different deposition angles.

Figure 2. AFM images of AlN thin films deposited at different incident angles. Column (I) 3D images, column (П) 2D images and column (Ш) fraction of void images.

Figure 3. XRD patterns of AlN films produced at different deposition angles.

Figure 4. Optical spectra of AlN thin films deposited at different oblique angles $(\alpha)$, and incident light angles $(\theta)$.

Figure 5. Refractive index of AlN thin films deposited at different oblique angles $(\alpha)$, and incident light angles $(\theta)$.

Figure 6. Optical band gap of AlN for films deposited at different angles $(\alpha)$, and different incident light angles $(\theta)$.

Figure 7. The dependence of energy gap of AlN thin films on the deposition angle.

Figure 8. Absorption spectra of AlN films deposited at different angles $(\alpha)$ and three different incident light angles $(\theta)$. 
Table 1. Comparison of the experimental growth angles and the results of tangent rule for different deposition angles of AlN films.

\begin{tabular}{ccc}
\hline $\begin{array}{c}\text { Deposition angle, } \\
\alpha\left(^{\circ}\right)\end{array}$ & Growth angle, $\beta\left(^{\circ}\right)$ & $\begin{array}{c}\text { Growth angle } \\
\text { (tangent rule) },\left(^{\circ}\right)\end{array}$ \\
\hline 0 & 0 & 0 \\
15 & 0 & 7.63 \\
30 & 15 & 16.10 \\
45 & 26 & 26.57 \\
60 & 30 & 40.89 \\
\hline
\end{tabular}

Table 2. Surface roughness, surface porosity and mean diameter of AlN thin films deposited at different angle.

\begin{tabular}{cccc}
\hline $\begin{array}{c}\text { Deposition angle, } \\
\alpha\left(^{\circ}\right)\end{array}$ & $\begin{array}{c}\text { Surface } \\
\text { roughness }(\mathrm{nm})\end{array}$ & $\begin{array}{c}\text { Surface porosity } \\
(\%)\end{array}$ & $\begin{array}{c}\text { Mean diameter } \\
(\mathrm{nm})\end{array}$ \\
\hline 0 & 1.7 & 7.93 & 26 \\
15 & 0.9 & 38.94 & 57 \\
30 & 4.3 & 40.20 & 46 \\
45 & 1.4 & 37.31 & 43 \\
60 & 2.0 & 39.52 & 33 \\
\hline
\end{tabular}



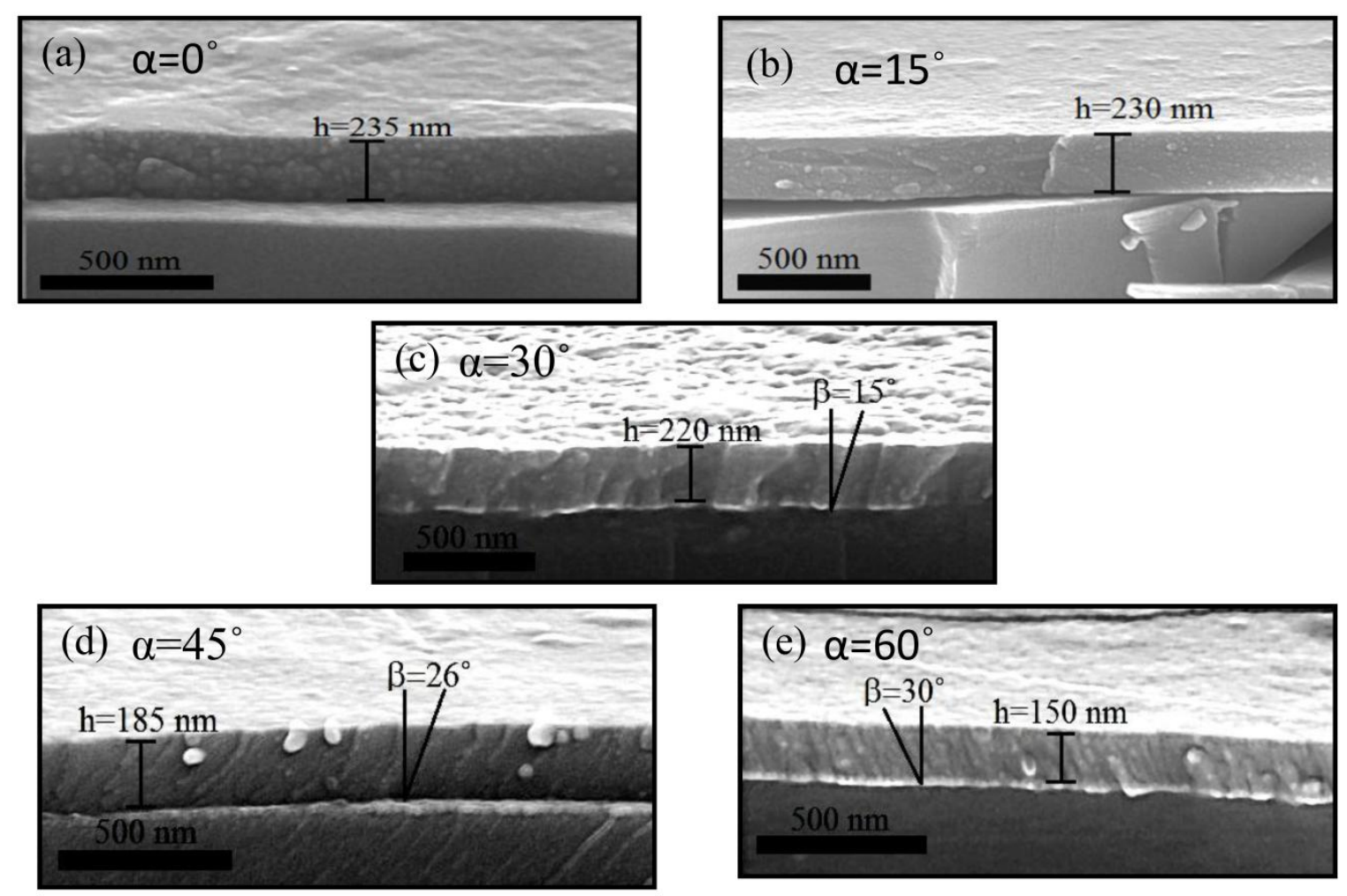

52.

Figure 1. FESEM images of AlN thin films with nano-rod structure produced at different deposition angles. 

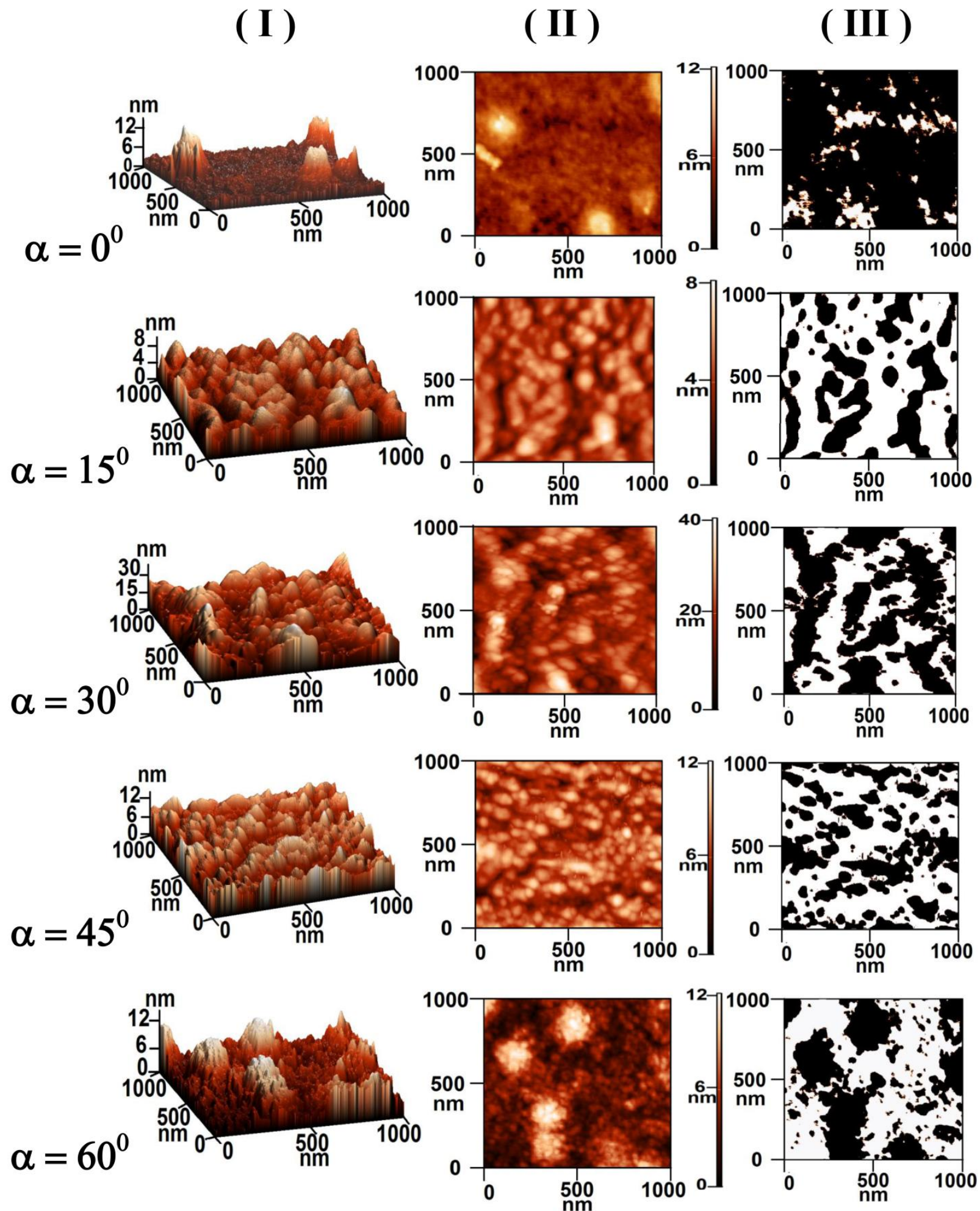

Figure 2. AFM images of AlN thin films deposited at different incident angles. Column (I) 3D images, column (П) 2D images and column (Ш) fraction of void images. 


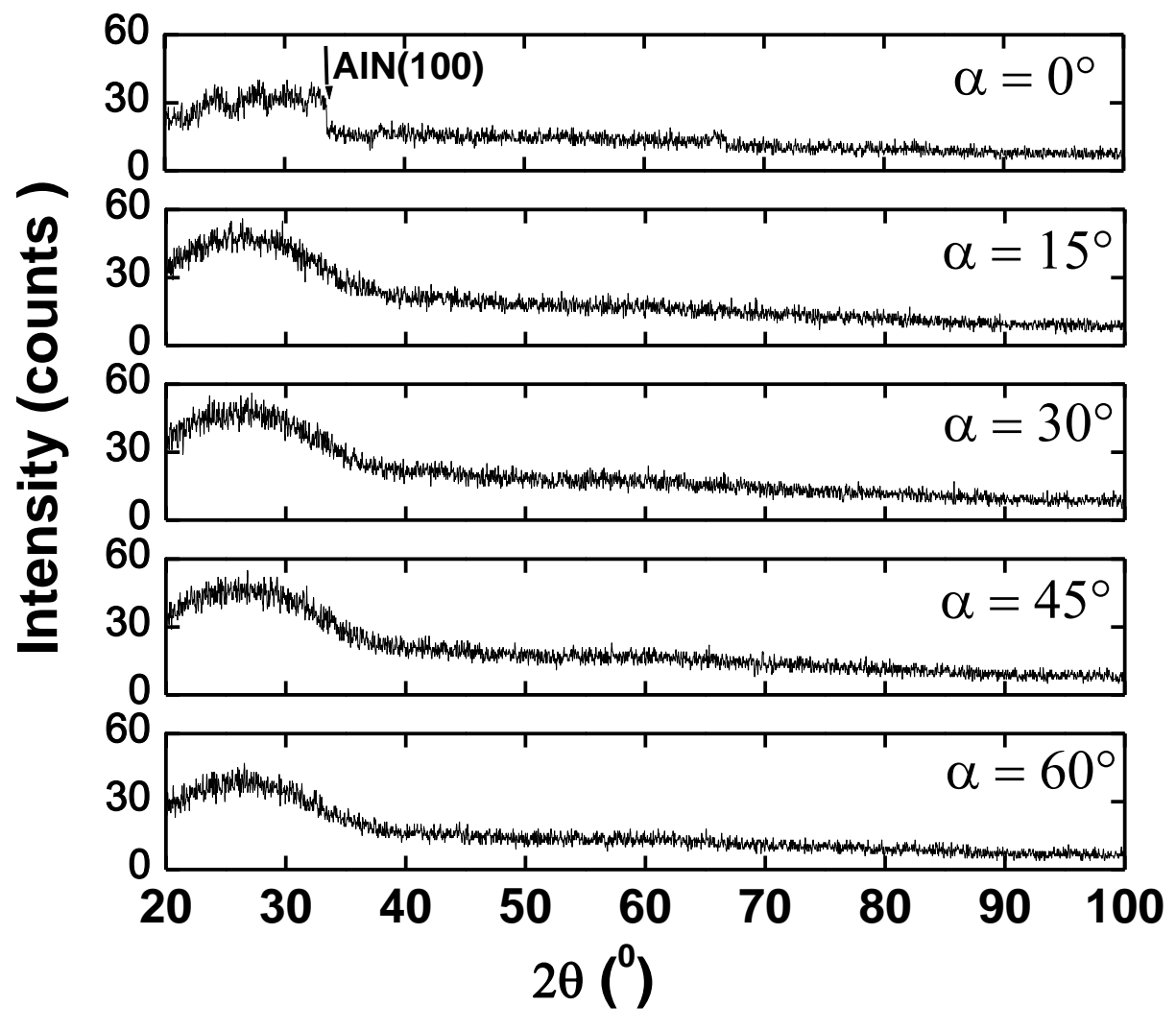

Figure 3. XRD patterns of AlN films produced at different deposition angles. 

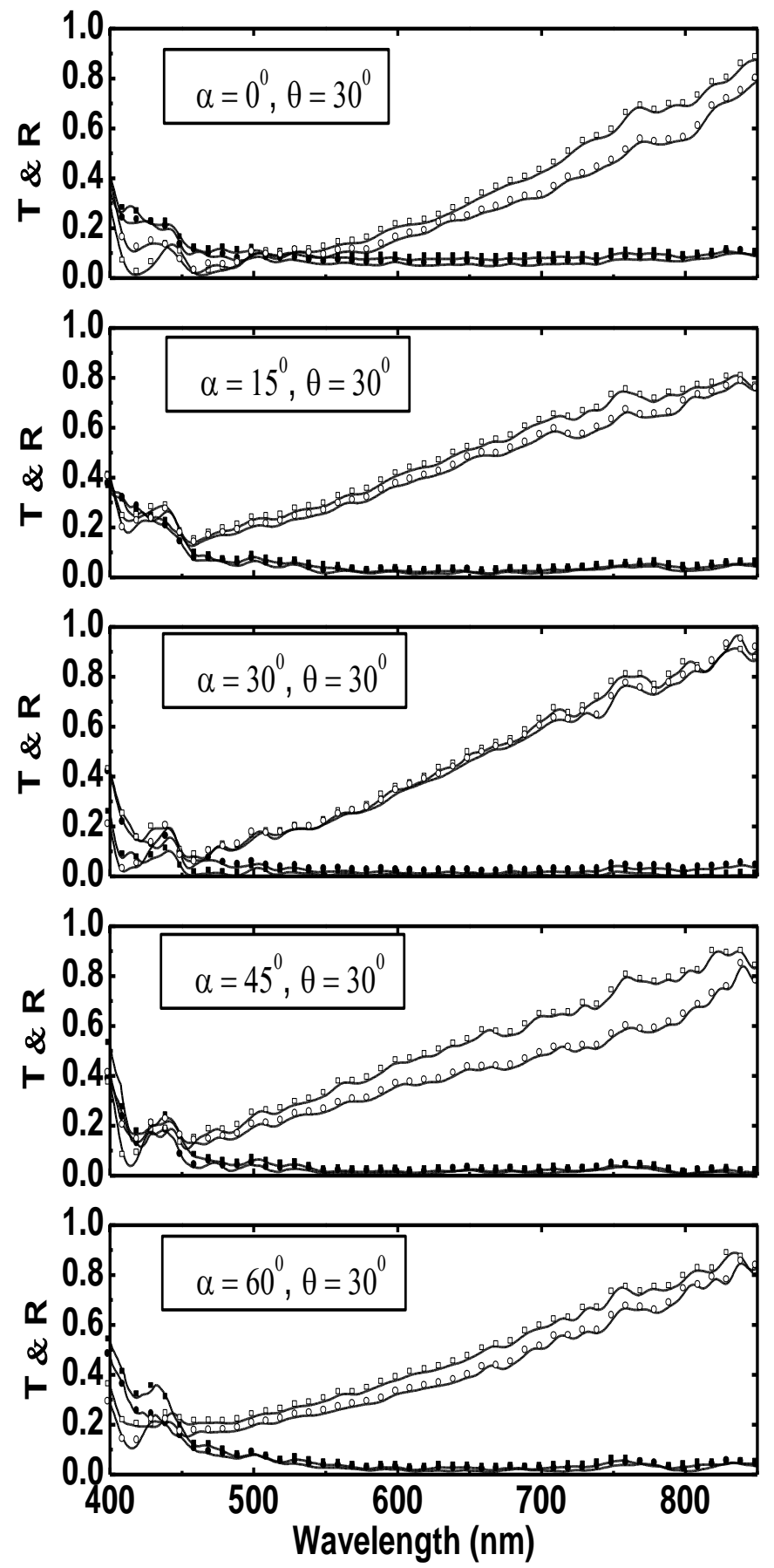
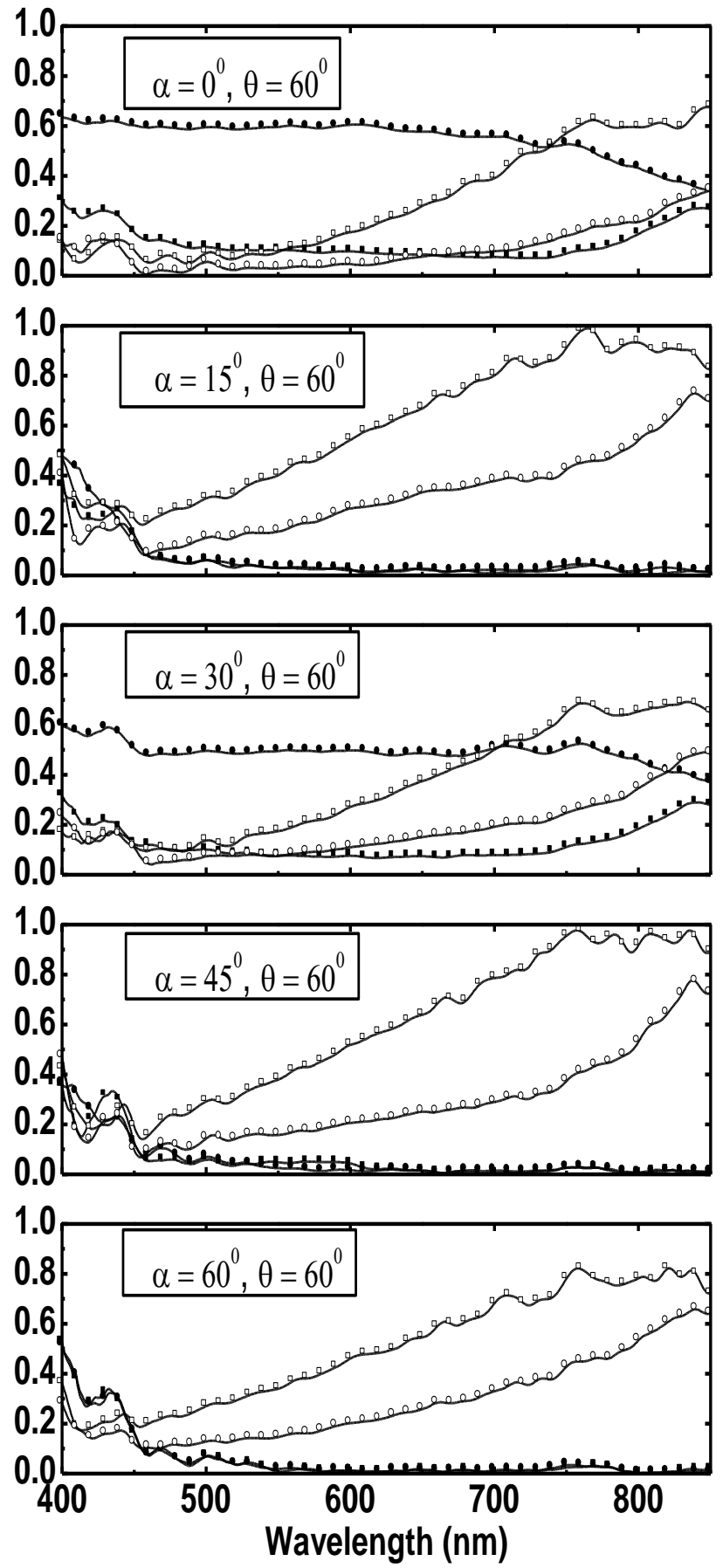

$$
\because R_{p}, \rightarrow R_{s}, \square-T_{p}, \multimap-T_{s}
$$

Figure 4. Optical spectra of AIN thin films deposited at different oblique angles $(\alpha)$, and for both sand p-polarized incident lights at two incident angles $(\theta)$. 

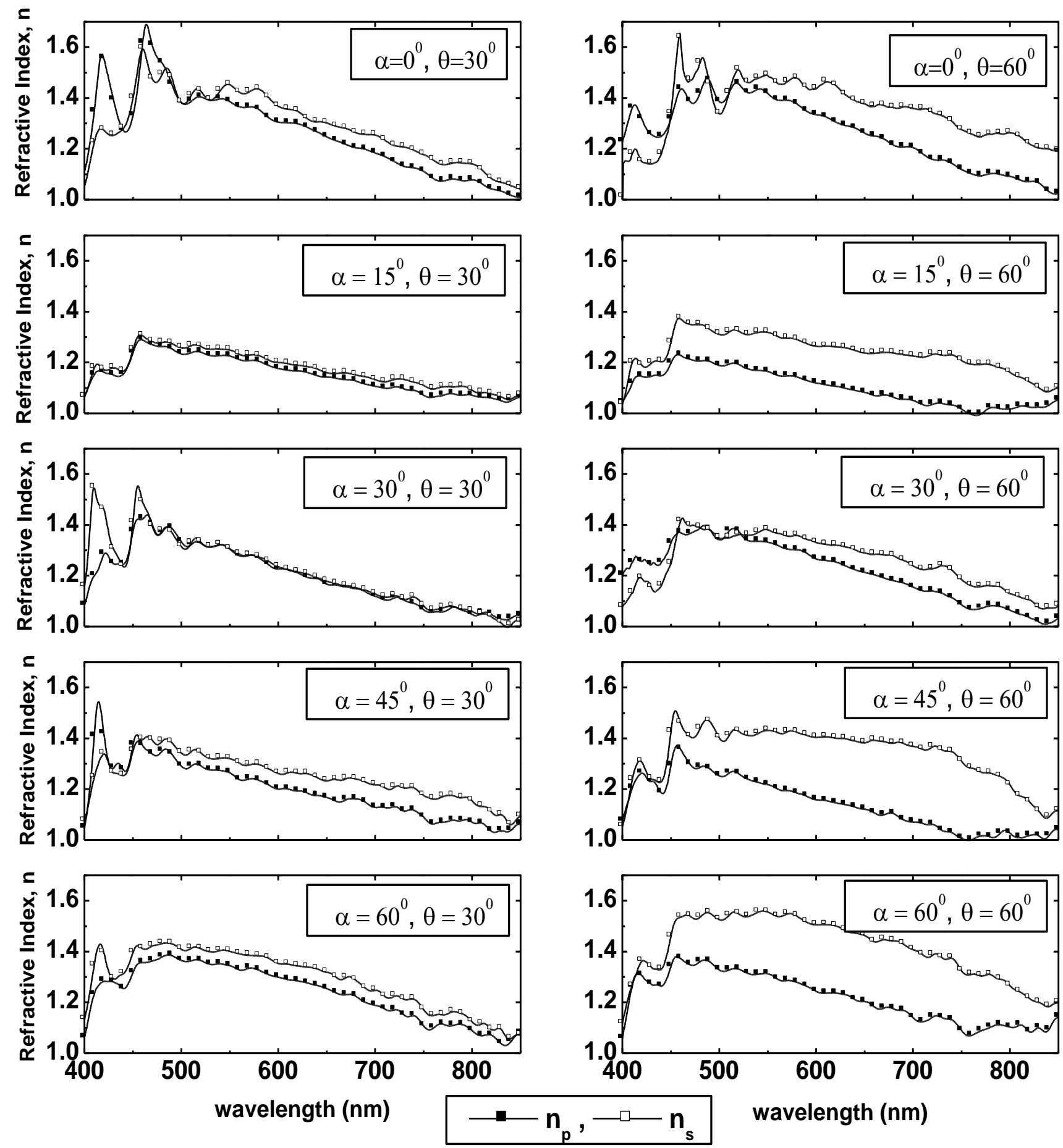

Figure 5. Refractive index of AlN thin films deposited at different oblique angles $(\alpha)$, and for both sand p-polarized incident lights at two incident angles $(\theta)$. 

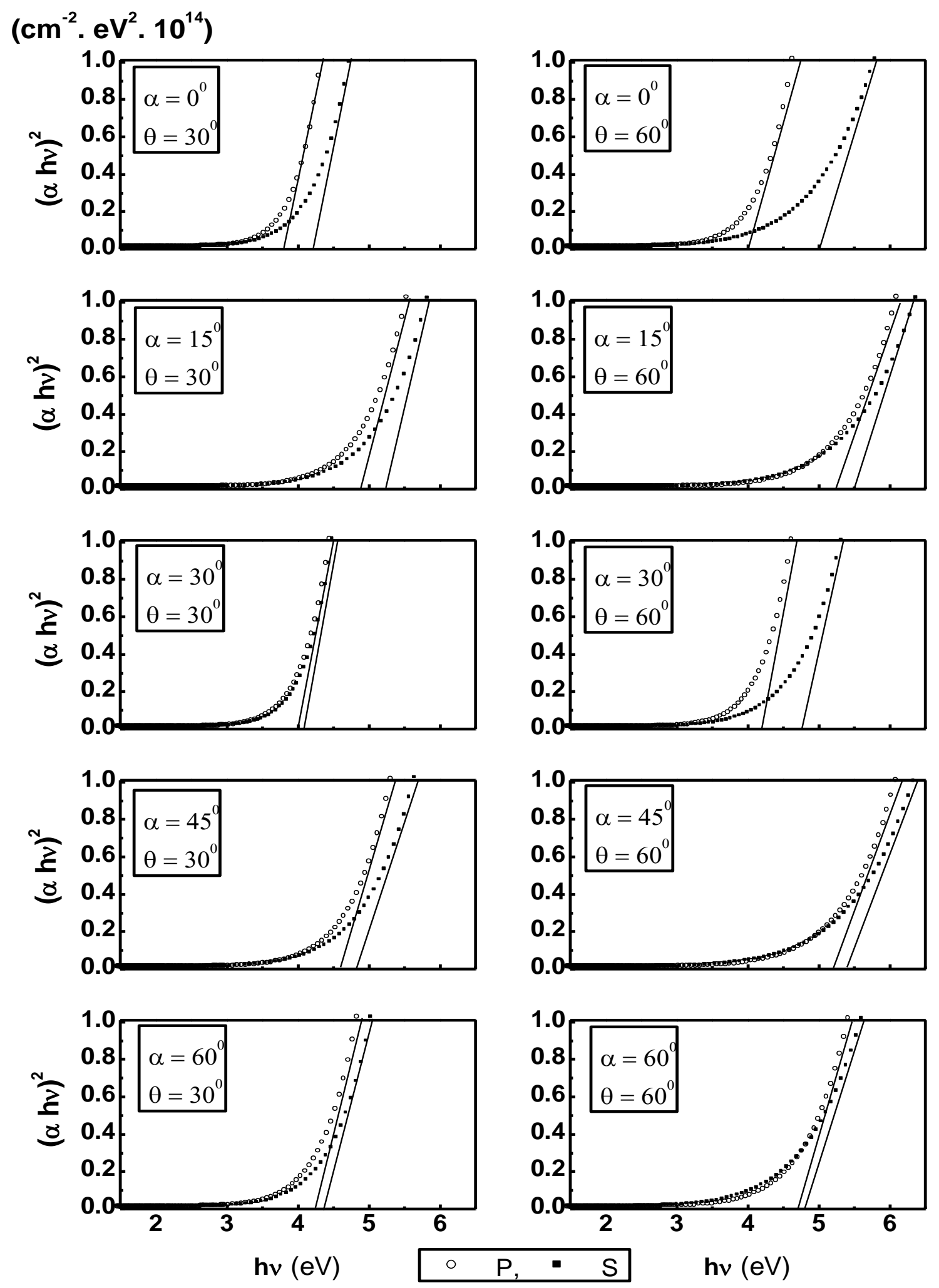

Figure 6. Optical band gap of AlN for films deposited at different angles $(\alpha)$, and different incident light angles $(\theta)$. 

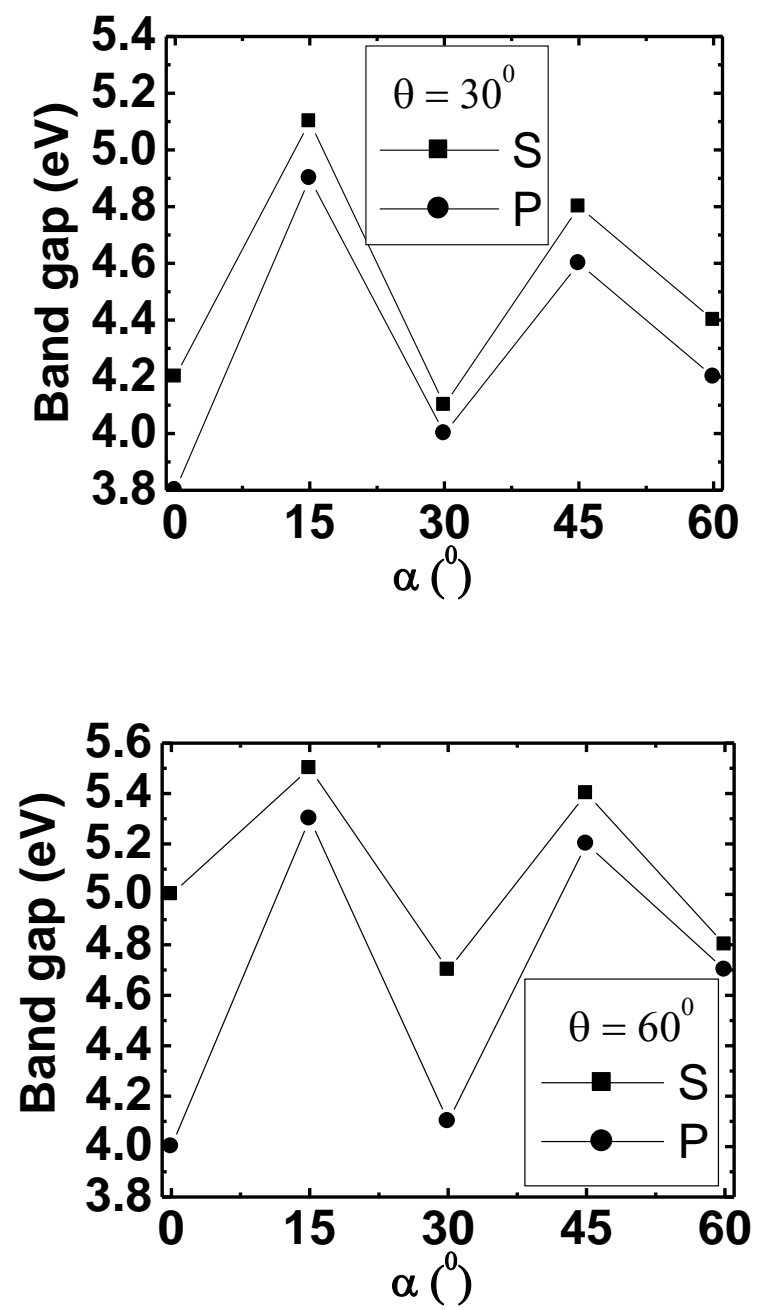

Figure 7. The dependence of energy gap of AlN thin films on the deposition angle. 

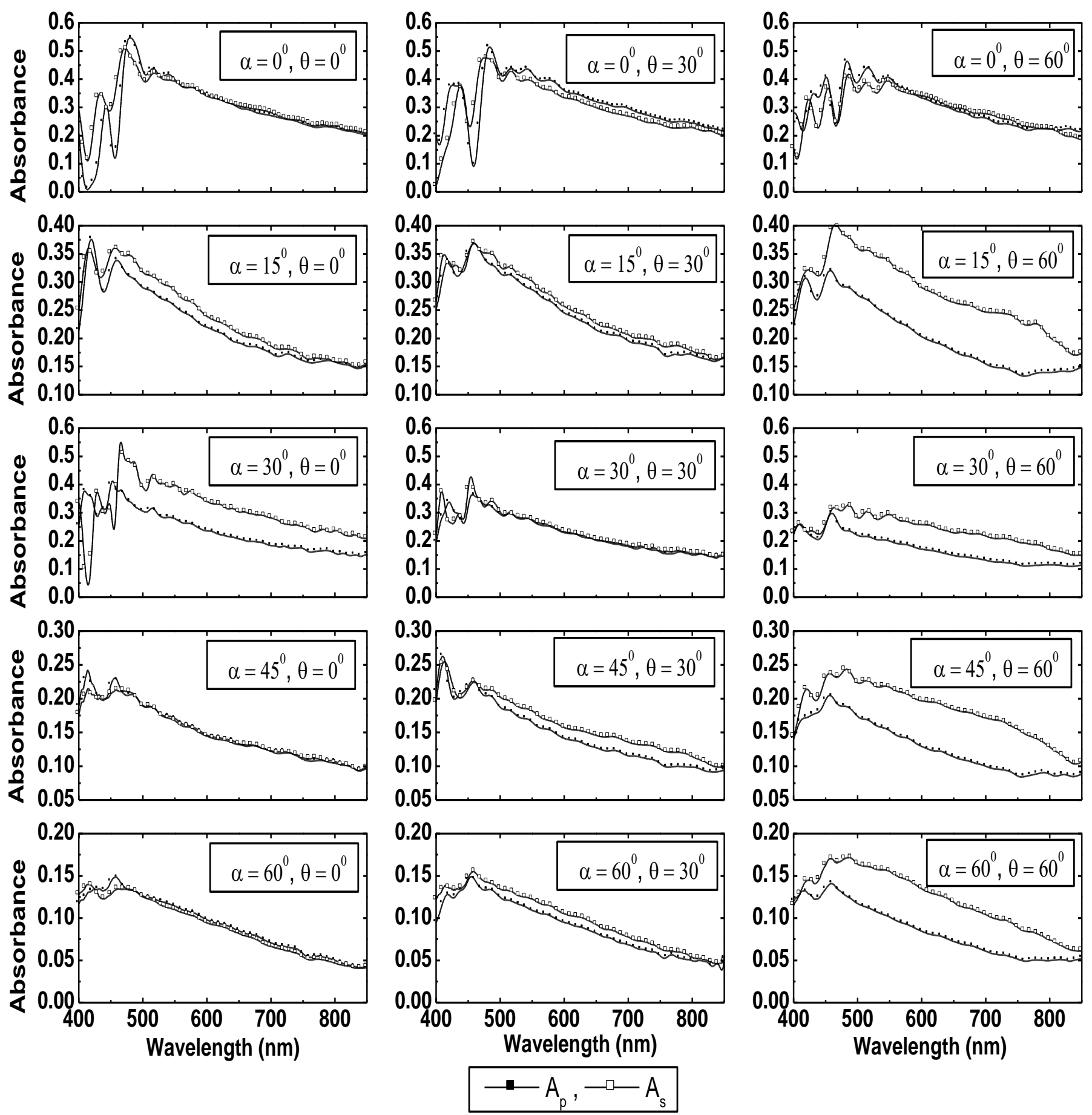

Figure 8. Absorption spectra of AlN films deposited at different angles $(\alpha)$ and three incident light angles $(\theta)$. 